\title{
Il titolo come specchio del testo: il caso delle 'Lettres écrites de Lausanne' e di 'Caliste, ou: suite des Lettres écrites de Lausanne'
}

\section{Paola Perazzolo}

\author{
(2) OpenEdition \\ Journals \\ Edizione digitale \\ URL: http://journals.openedition.org/studifrancesi/32642 \\ DOI: $10.4000 /$ studifrancesi.32642 \\ ISSN: 2421-5856

\section{Editore} \\ Rosenberg \& Sellier
}

\section{Edizione cartacea}

Data di pubblicazione: 1 décembre 2005

Paginazione: 486-500

ISSN: 0039-2944

\section{Notizia bibliografica digitale}

Paola Perazzolo, «ll titolo come specchio del testo: il caso delle 'Lettres écrites de Lausanne' e di 'Caliste, ou: suite des Lettres écrites de Lausanne'», Studi Francesi [Online], 147 (XLX | III) | 2005, online dal 30 novembre 2015, consultato il 20 avril 2021. URL: http://journals.openedition.org/studifrancesi/ 32642 ; DOl: https://doi.org/10.4000/studifrancesi.32642

\section{(c)}

Studi Francesi è distribuita con Licenza Creative Commons Attribuzione - Non commerciale - Non opere derivate 4.0 Internazionale. 


\section{Il titolo come specchio del testo: il caso delle 'Lettres écrites de Lausanne' e di 'Caliste, ou: suite des Lettres écrites de Lausanne'}

Secondo la doxa, il titolo è un elemento paratestuale che permette di distinguere un'opera da un'altra. Tale convinzione si rivela tuttavia poco precisa e niente affatto esauriente, anzi abbastanza limitata nella descrizione delle funzioni del titolo e dei rapporti che quest'ultimo intrattiene con tutti i suoi referenti. Nessuno può coscientemente sottostimare l'importanza del titolo di un'opera, letteraria o non. Eppure, le funzioni, gli effetti e il valore pragmatico di questo enunciato sono stati e restano tuttora abbastanza trascurati, abituati come siamo a considerare il titolo come «l'un de ces universaux littéraires dont l'existence va de soi» ${ }^{1}$. Solo negli ultimi trentacinque anni i critici hanno cominciato ad interessarsi seriamente a questo elemento, destinato a diventare in seguito uno degli aspetti paratestuali ${ }^{2}$ più analizzati. Gli studi di Léo Hoek, Gérard Genette e Charles Grivel, per non citare che alcuni nomi, hanno contribuito a questa presa di coscienza ${ }^{3}$, teorizzando statuto ${ }^{4}$, funzioni, implicazioni e suggestioni di un elemento fondamentale per una migliore comprensione dell'opera. L'oggetto di studio non è comunque dei più semplici, poiché il titolo si presenta ben più di altri enunciati come un vero e proprio «carrefour des codes et des genres» 5 .

Vera e propria presentazione di un'opera nella doppia accezione sottolineata da Genette $^{6}$, il titolo svolge presso il pubblico - da intendersi nella sua accezione più estesa $^{7}$ - una prima e importantissima funzione pragmatica, influenzando un eventuale acquisto e/o lettura. Paradossalmente, il primo approccio ad un'opera può even-

(1) M-H. Prat, Conclusions?, in A plus d'un titre. Les titres des ouvres dans la littérature française du Moyen Age au XXe siècle, Lyon, C.E.D.I.C., 2000, p. 232.

(2) Per la definizione di «elemento paratestuale» ci rifacciamo evidentemente a Gérard Genette: «L'œuvre littéraire consiste, exhaustivement ou essentiellement en un texte, c'est-à-dire (définition très minimale) en une suite plus ou moins longue d'énoncés verbaux plus ou moins pourvus de signification. Mais ce texte se présente rarement à l'état nu, sans le renfort et l'accompagnement d'un certain nombre de productions, elles-mêmes verbales ou non [...] dont on ne sait pas toujours si l'on doit ou non considérer qu'elles lui appartiennent, mais qui en tout cas l'entourent et le prolongent, précisément pour le présenter au sens habituel de ce verbe, mais aussi en son sens le plus fort: pour le rendre présent, pour assurer sa présence au monde, sa 'réception' et sa consommation, sous la forme, aujourd'hui du moins, d'un livre. Cet accompagnement, d'ampleur et d'allure variables, constitue ce que j'ai baptisé ailleurs [Palimpsestes] [...] le paratexte de l'œuvre. Le paratexte est donc pour nous ce par quoi un texte se fait livre et se propose comme tel à ses lecteurs, et plus généralement au public. 'Zone indécise' entre le dedans et le dehors, même sans limite rigoureuse [...] zone non seulement de transition, mais de transaction: lieu privilegié d'une pragmatique et d'une stratégie, d'une action sur le public au service, bien ou mal compris et accompli, d'un meilleur accueil du texte et d'une lecture plus pertinente», GENETTE, Seuils, Paris, Seuil, 1987, p. 7.

(3) Per la nostra analisi ci baseremo soprattutto su due importanti lavori: lo studio di L. HOEK, La marque du titre. Dispositifs sémiotiques d'une pratique textuelle, Paris-La Haye, Mouton, 1981 e il lavoro di GENETTE, Seuils, cit., pp. 59-106. Riprendiamo inoltre alcune considerazioni raccolte in $A$ plus d'un titre, cit., con particolare attenzione alla comunicazione di C. Fromilhague 'Titre principal' ou 'titre secondaire': la reformulation dans les titres, pp. 129-143.

(4) Non è nostra intenzione - né argomento della presente riflessione - addentrarci nella spinosa questione della definizione dello statuto del titolo, considerato talvolta come un «testo» in sé oppure qualificato come «para», «meta» o «pre» testo.

(5) M-H. PRAT, Conclusions?, in A plus d'un titre..., cit., p. 232.

(6) G. Genette, Seuils, cit., p. 7.

(7) Per Genette, il destinatario del titolo non è l'insieme dei lettori - a cui si rivolge invece il testo - ma il pubblico nella sua accezione più vasta. E infatti quest'ultimo che contribuisce alla circolazione e diffusione di un titolo, senza necessariamente averne letto il testo: «si le texte est un objet de lecture, le titre, comme d'ailleurs le nom de l'auteur, est un objet de circulation - ou, si l'on préfère, un sujet de conversation». Ibid., p. 79. 
tualmente prescindere dalla presenza fisica di quest'ultima, ma non dalla conoscenza dell'enunciato che la presenta. Motivazioni pragmatiche, oltre che estetiche ed artistiche, giustificano quindi il fatto che l'autore curi particolarmente la scelta del titolo, da considerare come un atto di comunicazione particolarmente ponderato, affatto secondario per la presentazione di un testo: «ce livre dont le titre dit tout», scriveva Baudelaire a proposito delle sue Fleurs du mal. Se la parola non è mai innocente, ci sembra che quelle che compongono il titolo lo siano meno di altre, anche a causa della densità e immediatezza di impatto di un enunciato di solito quantitativamente ridotto ma di grande importanza, visibilità e influenza grazie alla posizione liminare e strategica in cui si trova. Autonomo per posizione, tipografia, struttura sintattica e semantica e funzionamento, tale enunciato resta tuttavia dipendente dal suo referente primario, vale a dire il testo. Il titolo «apre» infatti il testo, di cui costituisce il primo elemento visibile, il più citato e diffuso, di cui programma e influenza la lettura, intrattenendo un rapporto paradigmatico con ogni frase dell'opera, che riassume almeno parzialmente ${ }^{8}$. L'enunciato assolve pertanto a tre funzioni principali: identifica in qualche modo l'opera a cui si riferisce, ne designa e ne valorizza il contenuto.

L'attenzione rivolta al titolo dall'autore - o, in alcuni casi, dall'editore, talvolta responsabile della scelta - non ha come unico scopo e conseguenza il concretizzarsi di una transazione commerciale. Atto di comunicazione immediato, l'enunciato influenza in modo primario la ricezione creando nel lettore attese che possono essere successivamente confermate o disattese, ma che non sono per questo meno presenti. Umberto Eco fa notare come i titoli più rispettosi del lettore siano quelli costituiti dal solo nome del protagonista poiché l'opacità del nome proprio, se privo di qualsiasi richiamo intertestuale, garantisce la neutralità semantica dell'enunciato ${ }^{10}$. Restando in qualche modo parzialmente «innocente», un simile titolo riduce al minimo la sua influenza sulla ricezione del testo. L'influenza dell'enunciato è in genere molto più importante: il titolo suggerisce, in modo più o meno esplicito ed evidente, elementi e chiavi di lettura del testo.

Il titolo di un'opera è altamente rivelatore. Non solo esso anticipa degli elementi della genesi o la lettura suggerita dall'autore, ma può indicare il periodo storico di appartenenza, e in alcuni casi anche l'autore, se questi adotta delle «manières titulaires» ${ }^{11}$ peculiari. Risultato di un processo socio-culturale, la produzione e ricezione di questo enunciato varia considerevolmente in base al contesto storico-culturale in cui l'opera è stata prodotta. Una specie di «historique» degli elementi del titolo evidenzia come in epoca classica quest'ultimo sia prevalentemente denotativo e relativamente non impostore. L'enunciato classico designa in genere elementi testuali e/o metatestuali e privilegia l'utilizzo dell'indicazione generica nel caso di opere teatrali o poetiche ${ }^{12}$. Successivamente, e soprattutto durante il XX secolo, i titoli diventano al tempo stesso più brevi e più ermetici, meno denotativi e più ambigui se non a volte completamente impostori. L'enunciato subisce insomma un processo di opacizzazione, processo reso più evidente anche dal progressivo abbandono dell'indicazione

(8) L. Hoek, op. cit., p. 2.

(9) Hoek precisa che il titolo è un «signal comportant un signifiant (suite de graphèmes), un signifié et un référent». Ibid., p. 27.

(10) U. Eco, Apostille au nom de la rose, Paris, Grasset, 1985, p. 510.

(11) GenetTe, Seuils, cit., p. 94.

(12) Genette fa notare come durante il periodo classico, i titoli di opere teatrali o poetiche siano ricchi di indicazioni generiche o parageneriche, mentre gli altri generi, non potendo esibire uno status altrettanto nobile, evitano di rivelarsi come tali. Durante il diciannovesimo secolo invece vige la moda dei titoli brevi, generalmente privi di indicazioni generiche. In questo periodo la menzione «roman» non è ancora considerata come abbastanza illustre per poter essere esibita, come invece accade nel ventesimo secolo, quando il genere narrativo è considerato più «vendeur» di altri. Per Genette l'innovazione delle indicazioni generiche è un tratto caratteristico del XX secolo. Ibid., pp. 97-98.

(13) Ibid., p. 89. 
generica. Anche il sottotitolo, che connota una costruzione prevalentemente classica, tende a sparire. Tutt'al più, la sua presenza rappresenta un vero e proprio «remède à l'amour» ${ }^{13}$ : dovendo chiarire il significato di un enunciato più volentieri «accrocheur» che trasparente ${ }^{14}$, il sottotitolo detiene una funzione esplicativa più che estetica.

Esistono dunque delle vere e proprie «manières titulaires» che caratterizzano e connotano un determinato autore o un periodo storico-letterario ben preciso ${ }^{15}$. Se il gusto contemporaneo per un titolo non strettamente denotativo implica una maggiore eterogeneità nella produzione, in epoca classica gli enunciati sono appunto più stereotipati. Essi sono infatti conformi ad una tradizione letteraria di chiarezza e di rispondenza titolo-testo, oltre che più orientati all'inserzione della menzione generica. Insomma, agli occhi di un lettore contemporaneo, molti enunciati classici appaiono quasi banali a causa della loro apparente semplicità o del loro alto livello di stereotipia. Questo giudizio può essere formulato anche a proposito di un buon numero di titoli narrativi settecenteschi, scarsamente originali perché rispondenti alle condizioni editoriali dell'epoca e a una poetica che condanna ancora il genere narrativo. La formula dei mémoires e della modalità epistolare, se deve il suo successo proprio all'aura di autenticità che impone al testo, suggerisce infatti durante tutto il secolo uno schema ripetuto e ripetitivo, comprensivo dell'indicazione paragenerica ${ }^{16}$ «Mémoires de...», «Histoire de...», «Lettres de...» e del riferimento, spesso abbastanza vago, all'elemento agente della diegesi. Le indicazioni di tali enunciati sono «generiche» in tutti i sensi: sia perché indicano il genere di appartenenza, sia perché sono vaghe, imprecise. A questo proposito la critica ha già insistito sull'assenza del termine «Roman» e sulla sua sostituzione con indicazioni che connotano l'opera come non fittizia, facendone una storia raccontata da un narratore che non si considera come tale e che comprensibilmente vuole restare anonimo. La genericità del termine risponde inoltre alla mancanza di termine dell'opera, esplicitando l'incompiutezza propria alla maggior parte dei testi settecenteschi. Jan Herman evidenzia come la sostituzione dell'indicazione generica «Roman» con quelle di «Histoire» e di «Mémoires» «connote une poétique narrative de l'inachevé, du désordre, du fragmentaire» ${ }^{17}$, poiché questi due termini veicolano una «forme d'inachèvement qui n'est plus accidentelle, mais qui s'inscrit au centre d'une nouvelle poétique du genre» ${ }^{18}$.

Tali considerazioni a proposito dell'indicazione generica sono interessanti poiché testimoniano di una realtà estetico-poetica estranea al lettore contemporaneo. La ricostruzione delle motivazioni non modifica in ogni caso il risultato finale: la maggior parte di questi titoli restano poco attraenti per un pubblico moderno. A meno di un agente fortemente connotato o dotato di una forte intertestualità, un enunciato che faccia parte della tradizione delle «Lettres de...» o dei «Mémoires de...» sembra

(14) Ibid.

(15) Genette richiama brevemente gli elementi distintivi degli enunciati delle diverse epoche: «il y a des connotations d'ordre historique: dignité classique des titres génériques, romantisme (et postromantisme) des titres paragénériques, couleur XVIIIe siècle des longs titres narratifs à la Defoe, tradition dix-neuviémiste des noms complets des héros, [...] titres-clichés des recueils surréalistes [...]. Il y a encore des connotations génériques: nom unique de héros en tragédie [...], nom de caractère en comédie [...], suffixe en -ade ou -ide des titres d'épopées classiques [...] qui conjoignent de manière très économique une indication thématique (par le nom) et une rhématique (par le suffixe), brutalité goguenarde des titres de série noire, etc.», ibid., p. 94.

(16) Genette precisa: «l'indication générique est une annexe du titre [...] par définition rhématique, puisque destinée à faire connaître le statut générique intentionnel de l'œuvre qui suit. Ce statut est officiel, en ce sens qu'il est celui que l'auteur et l'éditeur veulent attribuer au texte», ibid., p. 98.

(17) J. Herman, Topos de l'enfant abandonné et figures rhétoriques de l'inachèvement dans «Histoire d'une Grecque moderne» de Prévost, in A. RIVARA et G. Lavorel ed., L'ouvre inachevée, Actes du Colloque International, 11-12 décembre 1998, Lyon, CEDIC, 1999, p. 161.

(18) Ibid., p. 159. 
banale nella sua stereotipia, pienamente aderente al testo cui si riferisce e per ciò stesso poco seduttivo ${ }^{19}$. Il valore estetico di questi enunciati, così come il loro potere perlocutorio, non è tuttavia in discussione, poiché esso dev'essere ovviamente contestualizzato in base al suo periodo storico-culturale. Se oggi appare poco invitante, la formula succitata ha goduto di un indubbio successo durante tutto il XVIII secolo.

In ogni caso, il valore «accrocheur» e pragmatico di un titolo rappresenta solo uno dei suoi aspetti. Estetica a parte, questa formula settecentesca è veramente così scontata e banale quale ci appare oggi? In teoria, anche all'interno di una tradizione letteraria ben precisa, il titolo resta lo specchio di un testo singolo, cui si riferisce e che presenta. In altri termini, anche un enunciato apparentemente banale fornisce delle chiavi di lettura. Eco si rammaricava del fatto che un titolo costituisse già «sfortunatamente»- una «chiave interpretativa ${ }^{20}$. Se l'analisi dell'opera può - deve? - cominciare da quella del titolo, è forse possibile che tale enunciato, per stereotipato che sia, non rechi traccia dell'eventuale particolarità di un testo che, ricordiamolo, identifica, designa e valorizza?

A questo proposito, vorremmo provare a ritrovare nell'enunciato peculiarità e/o particolarità del testo. Il nostro ragionamento è induttivo, e quindi in qualche modo opposto al percorso «logico» di lettura precedentemente evocato. Partendo da opere che conosciamo e che si presentano come originali rispetto alla tradizione delle «Lettres de...» cui pertanto rimandano, vorremmo testare se e quanto il titolo, considerato come un atto comunicativo particolarmente espressivo, rifletta l'originalità della scrittura anche se si inserisce in una tradizione fortemente standardizzata.

Per rispondere a questi interrogativi, vorremmo prendere in considerazione $\mathrm{i}$ titoli di due romanzi di Isabelle de Charrière: le Lettres écrites de Lausanne (1785) e Caliste, ou: suite des Lettres écrites de Lausanne $(1787)^{21}$. Questi due enunciati presen-

(19) Secondo Genette, per il lettore contemporaneo il titolo dev'essere allusivo e intrigante piuttosto che esplicativo. Il suo valore pragmatico risiede dunque nella sua capacità di «opacification» piuttosto che di trasparenza. La produzione del titolo diventa allora una vera e propria strategia che riveste molteplici forme. Alcune di esse - quali l'allusione intertestuale, la riscrittura e/o la parodia, l'apparente assenza di coesione logica tra titolo secondario e principale - richiedono un'alta competenza culturale da parte del lettore. G. GENETTE, Seuils, cit., p. 87.

(20) Op. cit., p. 510.

(21) La forma dell'enunciato adottata è quella dell'edizione originale, quale appare in I. DE Charriere, Euvres complètes, Amsterdam, G. A. Van Oorschot, 1979-1984, vol. VIII, p. 615, note. Per questioni di comodità, designeremo i due titoli succitati come T1 e T2, e le due parti costituenti il T2 verranno indicate rispettivamente come $C$ e SLL. I due enunciati sono trattati singolarmente, ma poiché il secondo rimanda evidentemente al primo - che riprende in toto -, l'analisi di T1 è da considerarsi applicata e applicabile anche a una parte di T2, con le dovute eccezioni, che saranno debitamente indicate. Teniamo inoltre a sottolineare la dimensione di «oggetto artificiale» propria del titolo. Per la nostra applicazione, prenderemo in considerazione $\mathrm{i}$ soli T1 e T2, arbitrariamente astratti dalla pagina di titolo cui appartengono. Il che non ci impedisce di analizzare brevemente in questa sede alcuni aspetti di questo blocco ti- pografico quale ci viene presentato nelle note del volume VIII delle Euvres complètes. La pagina di titolo esprime la difficoltà espressiva tipica del romanzo dell'epoca. Colpisce la mancanza del nome dell'autore, procedimento impensabile in un testo contemporaneo ma usuale durante tutto il XVIII secolo. A quest'epoca il romanzo, genere considerato ancora come minore, non viene mai presentato come un'opera di finzione ma «spacciato» per una storia vera. L'adozione di questa strategia narrativa implica quindi l'assenza nella pagina di titolo di qualsiasi riferimento a una presenza autoriale o a uno status di opera narrativa. Anche il rimando all'editore connota il periodo al quale appartiene il libro. Nel caso della pagina di titolo di T2, la prolissità dell'indicazione contrasta con la brevità dei testi moderni, mentre la pagina di T1 non esplicita alcuna indicazione sull'editore, se non la menzione «Toulouse». Tale procedimento non è affatto inusuale. All'epoca, molti romanzi menzionavano indicazioni tipografiche ed editoriali false per aggirare censori e critici. Nel caso dei nostri due enunciati, la diversità di indicazione può essere facilmente spiegata: alla fine del secolo il genere narrativo ha già vinto la sua battaglia e non ha quasi più bisogno di tali sotterfugi. Inoltre, la prolissità della pagina di titolo di T2 è giustificata dal fatto che l'anonimato voluto da Madame de Charrière è stato facilmente svelato: già l'edizione successiva delle Lettres écrites de Lausanne, uscita un anno dopo quella originale, menziona editore e luogo di pubblicazione in modo quanto mai prolis- 
tano molteplici motivi di interesse: innanzitutto, soddisfano una prima condizione di base, cioè fanno riferimento a dei romanzi in cui l'autrice utilizza in modo originale le strutture e le formule tipiche dei «romans de femmes» settecenteschi. Anche se se ne distacca e in qualche modo la sovverte, Isabelle de Charrière si pone all'interno di una tradizione letteraria facilmente reperibile già dal titolo. Apparentemente, ci troviamo di fronte a due enunciati fortemente standardizzati, riconducibili alla tradizione letteraria delle «Lettres de...», tipiche di un periodo ben definito qual è quello del secolo dei Lumi. Tale stereotipia si manifesta non solo nell'utilizzo delle formule succitate o nell'indicazione dell'antroponimo femminile, ma anche nella ripresa del termine «suite», che testimonia di una prassi editoriale e di scrittura quanto mai frequente nel periodo preso in considerazione. Apparentemente banali a livello semantico e sintattico, i due enunciati che costituiscono il nostro corpus sono inoltre molto interessanti a livello teorico. In primo luogo per la loro struttura: T1 è infatti un titolo semplice e T2 un titolo doppio a riformulazione, il cui sottotitolo rimanda esplicitamente al primo enunciato, che riprende pari pari e che commenta. Successivamente, per la molteplicità delle relazioni che entrambi gli enunciati intrattengono con i loro diversi referenti a causa dei numerosi richiami intertestuali presenti. Tali rapporti si rivelano abbastanza problematici, evocando interrogativi che ci inducono a mettere in dubbio l'apparente semplicità di questi due titoli. Ad un esame più attento, si riscontrano infatti anomalie e contraddizioni semantiche e strutturali meritevoli di attenzione, in quanto entrambi gli enunciati si pongono in modo originale rispetto ad una tradizione letteraria alla quale apparentemente si conformano, così come fanno i testi cui si riferiscono. Questo parallelismo invita dunque a un esame più attento, per verificare se e quanto T1 e T2 si facciano veramente «specchio» dei testi di appartenenza.

Nella sua Postilla al nome della rosa, U. Eco scrive che un buon titolo deve «imbrigliare le idee, non imbrogliarle». Il gusto del lettore contemporaneo predilige senza dubbio l'ambiguità, il titolo ermetico e non immediatamente referenziale. Tuttavia, come abbiamo già accennato, il valore di seduzione di un enunciato non va giudicato solo in base a criteri astorici, ma dev'essere contestualizzato. E in ogni caso, ci sembra altrettanto interessante un enunciato che si faccia specchio dell'opera in modo ermetico. Ovvero, un titolo che, per quanto non «imbrogli» le idee, sia difficile da «imbrigliare». E, a nostro avviso, il caso dei due titoli in questione, che devono essere attentamente esaminati per essere compresi appieno e per rivelare la loro originalità. Esattamente speculari, in questo, ai testi cui si riferiscono, all'apparenza banali e composti da un insieme di «petits riens», dove il dettaglio conta molto e costituisce di fatto la chiave per ricomporre il tutto.

\section{T1: Lettres écrites de Lausanne}

Esaminato dal punto di vista linguistico, T1 è un enunciato fortemente stereotipato, sintatticamente semi-grammaticale e caratterizzato da uno stile ellittico e nominale, stile che contraddistingue la maggior parte dei titoli ${ }^{22}$. In questo caso, l'irregolarità sintattica è originata dal carattere ellittico dell'enunciato, che resta comunque pienamente accettabile: la soppressione della copula e l'utilizzo del predicato - il verbo écrire - con valore di aggettivo non comportano infatti alcuna irregolarità semantica. L'enunciato è anzi reso più assertivo, conciso e stereotipato.

so. Tipica dell'epoca classica è anche l'inserzione di un qualche ornamento tipografico nella pagina di titolo, evocato ma non meglio precisato dalla descrizione offerta nelle note a p. 615 del volume VIII delle Euvres complètes.

(22) L. Hoek, op. cit., p. 54. 
La forte stereotipia propria ai titoli permette dunque al lettore di catalogare questo enunciato come una frase completa e accettabile a livello di competenza discorsiva se non linguistica ${ }^{23}$. T1 si presenta come fortemente standardizzato anche dal punto di vista sintattico, l'uso del sintagma nominale essendo segnalato da Hoek come una caratteristica della costruzione dei titoli ${ }^{24}$. In questo caso, l'enunciato è composto da un nome comune plurale privo di predeterminante, da un participio passato con valore aggettivante e da un genitivo locativo indicante il luogo di provenienza. L'assenza di qualsiasi predeterminante contribuisce ad astrarre l'enunciato conferendogli una maggiore densità e una dimensione più generale.

A livello logico-semantico, T1 è senza dubbio un enunciato denotativo, che anticipa correttamente sia la forma che il contenuto del testo cui si riferisce. T1 informa e nasconde al tempo stesso, mantenendo la sua ambiguità. Esso esplicita anche tutte le funzioni proprie al titolo - identifica, designa e valorizza l'opera -, ma non può essere considerato come esclusivamente e puramente «denotativo». Riprendendo la tipologia di Genette, T1 è infatti un titolo misto, poiché unisce in un solo enunciato due elementi chiaramente separati, l'uno rematico («Lettres») e l'altro tematico («écrites de Lausanne»), assolvendo quindi in modo diverso e concorrenziale la funzione descrittiva. L'enunciato designa infatti il testo cui fa riferimento, ma non ne indica tanto o solo il «contenuto» quanto, piuttosto, la «forma». Gli elementi che costituiscono T1 rimandano al testo in modo letterale e non simbolico, suggerendo rispettivamente il tipo di diegesi e l'elemento spaziale. «Lausanne» è infatti un elemento tematico di determinazione topologica poiché anticipa un elemento della diegesi - il luogo di composizione e di provenienza, che può essere teoricamente distinto da quello di svolgimento degli eventi -, mentre «Lettres» indica il tipo di diegesi. I due elementi - rispettivamente rematico e tematico - sono collegati tra loro da «écrites de», un operatore proposizionale che indica la provenienza, in questo caso geografica.

A questo proposito, ci sembra interessante riprendere la precisazione effettuata da Genette a proposito dell'imprecisione della sua definizione di elemento «temati$c o\rangle^{25}$, imprecisione che si può effettivamente riscontrare anche nel caso di T1. Risulta infatti difficile considerare «Lausanne» come un «tema», mentre appare più esatto valutarlo come un elemento anticipatore della diegesi fortemente connesso al tema. In effetti, il romanzo tratta della città svizzera, di cui offre una descrizione sociale e di costume se non prettamente geografica, senza per questo essere un romanzo su Losanna. Per quanto a prima vista impreciso, l'aggettivo «tematico» è tuttavia in questo caso fondamentalmente appropriato per la descrizione dell'anomalia evidente in T1 rispetto ad altri enunciati suoi contemporanei. In T1 il locutore focalizza l'attenzione del lettore sull'elemento spaziale più che sull'agente della diegesi, e lo fa apparentemente a discapito del potere «accrocheur», seduttivo, dell'enunciato. Gli elementi denotativi presenti non sono accompagnati da alcun predicato positivizzante o negativizzante e non assumono alcuna connotazione morale. Ma queste «lettres»

(23) Le trasformazioni operate sono recuperabili, poiché il lettore può ricostruire facilmente la struttura soggiacente, in questo caso $\mathrm{P}$ titolo $->$ PROP SN1 SN2. L'enunciato regolare e completo sarebbe: «Le/Ce livre est intitulé Lettres [qui sont/ont été] écrites de Lausanne». La presenza di una struttura profonda grammaticalmente regolare consente di definire T1 come una frase semi-grammaticale semanticamente accettabile.

(24) L. HoEk, op. cit., p. 64.

(25) Genette sottolinea il fatto che l'uso del termine «tematico» sarebbe improprio poiché presuppone un «élargissement que l'on peut juger abusif de la notion de thème» in quanto«un lieu (tardif ou non), un objet (symbolique ou non) un leitmotiv, un personnage, même central, ne sont pas à proprement parler des thèmes, mais des éléments de l'univers diégétique des œuvres qu'ils servent à intituler». Il critico mantiene tuttavia la sua definizione, da intendersi come una «synecdoque généralisante qui sera, si l'on veut, un hommage à l'importance du thème dans le 'contenu' d'une œuvre», come un elemento che rinvia al contenuto è comunque in relazione con il tema. G. GENETTE, Seuils, cit., p. 85. 
sono socialmente connotate dal rimando a Losanna, una città relativamente piccola e provinciale, molto poco romanesque.

L'inserzione dell'elemento geografico nell'enunciato risulta in effetti problematica. Non solo il rimando ad un'ambientazione geograficamente poco quotata qual è quella rappresentata dalla Svizzera ${ }^{26}$ riduce sensibilmente il fascino di T1, ma esso rappresenta anche un'anomalia rispetto alla tradizione del romanzo epistolare nella quale l'opera si inserisce. Si può infatti ritrovare facilmente nella formula «Lettres [...] de...» una citazione esterna rivolta al genere epistolare. Tale intertitolarità implicita, per riprendere $\mathrm{i}$ termini di Hoek ${ }^{27}$, testimonia di una tradizione letteraria precisa, poiché connota un genere di scrittura e un periodo letterario ben definito - il XVIII secolo. La scelta della dimensione geografica piuttosto che l'inserzione di un complemento di agente sembra tuttavia leggermente discordante rispetto al modello succitato. L'assenza di un antroponimo o di un rimando a un nome di finzione, proprio o meno, - secondo la tradizione delle «Lettres de la Marquise de...» ecc. - e la menzione di un elemento spaziale «alternativo» indicano infatti l'importanza accordata alla dimensione geografica a scapito di quella individuale, ribadita tuttavia dalla modalità epistolare. Se l'elemento rematico «Lettres» rimanda al romanzo sentimentale, imperniato sulla descrizione dei moti dell'animo più che su quella topografica, l'elemento geografico rende dubbia e più difficile la classificazione generica dell'opera. L'ambiguità originata in T1 dall'inserzione dell'operatore «écrites de...» corrisponde all'ambiguità intrinseca del testo. L'elemento preposizionale è infatti bivalente, poiché evoca in modo diretto il luogo di composizione e in modo indiretto la presenza di un agente implicito: le lettere sono «écrites de Lausanne» implicitamente da qualcuno e non sono, in modo più esplicito ma anche più riduttivo, semplicemente «lausannoises». Questa anomalia richiama la bipolarità del testo di Madame de Charrière, che si situa in effetti a metà tra romanzo sentimentale e «roman de mœurs». In T1, la «tematizzazione» dell'elemento spaziale annuncia quindi l'ambizione descrittiva e «realista ${ }^{28}$ che sarà propria del romanzo, nonché l'importanza che questo aspetto assume nella scrittura dell'autrice ${ }^{29}$.

Nonostante questa singolarità, T1 rimanda comunque ad un testo classico, soprattutto per la menzione all'elemento «Lettres», evidenziato a livello morfologico dall'allitterazione delle lettere «l», «t», «r» e a livello sintattico da un'antonomasia generalizzante. In T1, entrambe le figure hanno la funzione di porre l'accento sul termine rematico, che acquista importanza e la cui estensione è resa perciò più densa. A livello metanarrativo, «Lettres» acquisisce infatti un valore forte, accrescendo il potere perlocutorio dell'enunciato. Come abbiamo già accennato, nella prima metà, e comunque durante tutto il XVIII secolo, la formula «Lettres» è tra le più usate per creare un'illusione di verità, il che ne accresce presso il pubblico dell'epoca il potere di seduzione. Il valore seduttivo di T1 è aumentato dall'uso del termine rematico, la cui connotazione di realtà è ulteriormente incrementata dalla funzione generaliz-

(26) Fanno eccezione le ambientazioni alpine, il cui successo si situa sulla falsariga della Nouvelle Héloïse. Il décor scelto da Madame de Charrière è tuttavia urbano e non ha nulla a che vedere con la maestosità dei paesaggi descritti da Rousseau.

(27) L. Hoek, op. cit., p. 184.

(28) Il termine «realismo» è senza dubbio da usarsi con prudenza a proposito di un testo del XVIII secolo. Tuttavia, per le sue ambientazioni mediocri, l'attenzione ai dettagli e agli avvenimenti dell'esistenza quotidiana, l'assenza di avvenimenti o passioni troppo «romanesques» la critica ha spesso utilizzato questo termine a proposito dei primi ro- manzi di Madame de Charrière.

(29) Appena un anno prima della pubblicazione delle Lettres écrites de Lausanne, Madame de Charrière confessava in una lettera a un amico il suo interesse per una scrittura «realista», e l'influenza esercitata sulla composizione delle Lettres Neuchâteloises da un romanzo olandese pubblicato nel 1782, l'Histoire de Sarah Burgerhart: «Je venois de voir dans Sara Burgerbart (roman hollandais) qu'en peignant des lieux et des mœurs que l'on connaît bien, l'on donne à des personnages fictifs une réalité précieuse» I. DE CHARRIÈRE, Euvres complètes, cit., vol. VI, p. 558. 
zante attribuita all'uso del plurale, dalla soppressione dell'articolo e dalla menzione di un elemento spaziale ben preciso. La veridicità di T1 inteso come atto illocutorio è dunque accresciuta dall'uso del termine generico «Lettres», così come dalle circostanze relative alla sua «nascita» ${ }^{30}$. Genette evidenzia infatti l'importanza della «vie prénatale» di un enunciato, poiché le condizioni di composizione possono fornire informazioni interessanti a proposito delle intenzioni dell'autore ${ }^{31}$. In questo caso, è degna di attenzione l'assoluta mancanza di «vita prenatale» di T1. Secondo la corrispondenza di Madame de Charrière, sarebbe l'enunciato che avrebbe funzionato come incitateur per la composizione del testo e non viceversa ${ }^{32}$. T1 sarebbe quindi stato concepito di slancio e considerato già «perfetto», senza bisogno di nessuna ulteriore modifica.

\section{T2: Caliste, ou: suite des Lettres écrites de Lausanne}

A livello linguistico, anche T2 si presenta come un enunciato semi-grammaticale pienamente accettabile dal punto di vista semantico e caratterizzato da uno stile ellittico e nominale. T2 è composto da un titolo principale - un nome proprio di finzione - e da un titolo secondario - una frase nominale. Dal punto di vista sintattico, è facile determinare che $C$ è il titolo principale e $S L L$ il titolo secondario. I due enunciati sono infatti separati da indici tipografici - la virgola e i due punti - e collegati da una congiunzione - «ou» -, il cui valore di coordinazione è superiore a quello di separazione veicolato dai due punti, che disgiungono chiaramente le due parti dell'enunciato.

A livello semantico, il nome proprio di finzione «Caliste», segnalato dalla capitalizzazione e dall'assenza di articolo, è un elemento tematico che indica l'agente della diegesi; «suite» è invece un elemento rematico e intertestuale che si pone a un livello metanarrativo e che assume un valore connotativo positivizzante. Per quanto concerne i rapporti semantici tra titolo principale e titolo secondario, la congiunzione disgiuntiva «ou» permette la citazione dell'elemento agente da parte del titolo secondario. A livello morfo-sintattico, il morfema «ou» esprime una congiunzione inclusiva, collegando quindi due titoli «co-validables», per riprendere la terminologia usata da Catherine Fromilhague nel suo studio sugli enunciati a riformulazione ${ }^{33}$ : poiché $C$ e SLL sono ugualmente veri e l'uno non esclude l'altro, T2 è dunque un titolo a doppia denominazione. Se due unità sono «co-validables», l'una sostituisce l'altra - SLL funge da parafrasi esplicativa di $C$ - ed entrambe si pongono allo stesso livello linguistico.

(30) T1, contrariamente a T2, non sarà mai modificato né ridotto dal 1785 , anno di uscita dell'edizione originale e data di nascita dell'enunciato in questione.

(31) G. Genette, Seuils, cit., p. 70.

(32) All'inizio del 1785 , uno sconosciuto annuncia l'imminente pubblicazione di un'opera intitolata Lettres lausannoises. La notizia provoca una forte reazione da parte di Isabelle de Charrière, che racconta così l'episodio in una lettera del 1804: «Un an après que l'on eut imprimé les Lettres Neuchâteloises, un proposant du Pays de Vaud publia dans un prospectus trois volumes des Lettres lausannoises. Il annonçait les plus belles choses du monde, mais il voulait une souscription. 'Quoi, dis-je, on me vole mon titre! Mais je préviendrai ce pédant audacieux.' - Aussitôt je montai dans ma chambre et me dépêchai d'écrire. Huit ou dix jours après, les Lettres écrites de Lausanne étaient faites», I. De Charrière, CEuvres complètes, cit., vol. VI, p. 559. Composto e rivisto nel gennaio del 1785, il testo appare nella primavera dello stesso anno.

(33) C. Fromilhague, 'Titre principal' ou 'titre secondaire'..., in A plus d'un titre..., cit., p. 130. Essendo l'unico elemento di riformulazione che riesce a suggerire nello stesso tempo una differenza metalinguistica e un'equivalenza, solo la congiunzione «ou» potrà essere usata. In effetti, sostituendovi altri elementi di riformulazione quali «c'est-à-dire» o «en d'autres termes», T2 è percepito come anomalo. L'assenza di un legame logico-semantico tra le due parti dell'enunciato non tollera l'imposizione di un'equivalenza - solo suggerita da «ou»-, quale potrebbe essere in Caliste: en d'autres termes, Lettres écrites de Lausanne. 
A livello logico-semantico, T2 è un enunciato che mescola elementi connotativi e denotativi: anche a causa dell'assenza di un legame logico tra le due parti dell'enunciato, esso necessita di uno sforzo interpretativo da parte del lettore. Il titolo in questione è ambiguo e parzialmente impostore, poiché il testo è solo in parte una continuazione delle Lettres écrites de Lausanne, di cui non porta sicuramente a compimento la diegesi. Nel testo, Madame de Charrière, giustappone l'«histoire de Caliste» a quella di Cécile, l'eroina del romanzo precedente. L'accostamento del titolo principale e di quello secondario rivela quindi efficacemente le due «anime» dell'opera cui fa riferimento, svelando tuttavia quale sia la vicenda prioritaria: la posizione privilegiata accordata all'antroponimo «Caliste» e l'assenza di qualsiasi menzione al nome di Cécile esplicita la preminenza accordata dall'autrice alla prima storia. Per quanto le due storie risultino strettamente legate a livello tematico, l'esclusione dell'elemento «lausannois» sarà recepita anche dal pubblico, come testimonia la vera e propria «erosione» ${ }^{34}$ subita da T2 nel tempo. Nella corrispondenza della stessa Madame de Charrière ${ }^{35}$ così come in seguito, il titolo secondario verrà spesso omesso, sottolineando in tal modo come il testo sia l' «histoire de Caliste» assai più che la «suite» di quella di Cécile. Come ricorda Genette, la scelta, inizialmente motivata, di un enunciato da parte di un autore assume successivamente un mero valore convenzionale e identificativo. Il titolo può quindi arbitrariamente essere mutato a patto che sia preservato il suo valore di identificazione ${ }^{36}$. In questo caso il pubblico, che ne è in ultima istanza e alla lunga il vero artefice, ha successivamente optato per una più pratica erosione del sottotitolo, percepito come facoltativo ${ }^{37}$. Tale erosione, abbastanza comune, non è comunque ininfluente a livello di significato poiché risulta semanticamente limitata e limitante. In questo caso, non solo essa rende l'enunciato meno efficace a livello identificativo - se T2 assolve pienamente e in modo non equivoco questa funzione, il solo Caliste è più ambiguo, esistendo altri testi omonimi, come vedremo -, ma elimina anche qualsiasi richiamo intertestuale all'opera precedente di Madame de Charrière, con cui il testo mantiene invece una relazione strettissima.

I rapporti tra le due storie e, per estensione, tra le due opere, sono in effetti alquanto ambigui ma molto forti, come risulta chiaramente dall'enunciato originale. $\mathrm{T} 1$, così com'è stato originariamente concepito, evoca pienamente l'indeterminatezza del legame esistente tra i due romanzi, offrendone al tempo stesso anche una chiave di lettura. In Seuils, G. Genette evidenzia quanto sia importante considerare il titolo quale appare al momento della sua «nascita», cioè all'uscita dell'edizione originale. Questo criterio serve ad evitare malintesi dovuti a modificazioni successive operate non tanto dall'autore quanto dal pubblico o dall'editore, che impongono così un'interpretazione strettamente personale e a volte non rispondente alla volontà prima dell'artista. Come abbiamo visto, questo è accaduto anche nel caso di $\mathrm{T} 2$, progressivamente «eroso» e reso autonomo da qualsiasi richiamo intertestuale e/o legame con le Lettres écrites de Lausanne. L'eliminazione del sottotitolo non costituisce comunque l'unica variazione subita da T2: le edizioni successive rivelano infatti ulteriori modifiche, apparentemente secondarie ma il cui esame non è privo

(34) G. Genette , Seuils, cit., p. 74.

(35) «Caliste ne fut écrite qu'assez longtemps après, c'est-à-dire un an peut-être[...] j'ai glissé sur Caliste ...» (Lettre de janvier-février 1804 à Taets van Amerongen); «M. Prault convint avec M. Suard qu'il imprimera Caliste, aussi bien que [...] Caliste avait eu un grand débit [...] j’avais gardé le plus grand silence sur Caliste pendant plusieurs mois», citato nell'introduzione alle Lettres écrites de Lausanne in I. De Charriere, Euvres complètes, cit., vol. VIII, pp. 125, 130.

(36) G. GenETte, Seuils, cit., p. 84.

(37) «Le principal agent de la dérive titulaire n'est probablement ni l'auteur, ni même l'éditeur, mais bien le public, et plus précisément [...] la postérité. Son travail - ou plutôt, en l'occurrence, sa paresse - va généralement dans le sens d'un raccourcissement, d'une véritable érosion du titre», ibid., p. 74. 
di interesse per meglio comprendere com'è stata recepita l'opera. In alcune edizioni settecentesche si riscontra la scomparsa del segno tipografico - i due punti e/o la virgola - e un'esitazione tra le indicazioni «suite»e «continuation» ${ }^{38}$. A un livello immediato, eliminare il segno tipografico significa indebolire la divisione tra le due parti del titolo; a un livello più inconscio, significa minare la separazione tra le due opere di Madame de Charrière. L'esistenza o meno del segno tipografico rafforza o indebolisce dunque l'espressione del rapporto di dipendenza tra i due testi. Anche la modifica dell'indicazione paragenerica non è semanticamente neutra. In Palimpsestes, Genette ha precisato la distinzione, funzionale e strutturale, che esiste tra «suite» e «continuation». Citando il Dictionnaire des synonymes di D'Alembert, secondo cui «on donne la continuation de l'ouvrage d'un autre et la suite du sien» e il Littré, che considera «suite» un termine «plus général, n'impliquant pas que ce à quoi on donne une suite soit ou non achevé, au lieu que continuation exprime positivement que la chose était restée à un certain point qui ne la terminait pas», Genette fa notare come la «continuation» e la «suite» siano rispettivamente una produzione allografa o autografa ${ }^{39}$. La menzione «suite», presente nell'edizione originale, sarebbe dunque teoricamente corretta, non solo poiché entrambi i testi sono di Isabelle de Charrière, ma anche perché resta più vaga circa la compiutezza o meno delle opere. Al contrario, le modifiche operate successivamente in T2 risultano leggermente scorrette perché fuorvianti. L'esitazione tra i due termini suggerirebbe un'incertezza nell'attribuzione della paternità dei romanzi. Nel caso delle Lettres écrites de Lausanne e di Caliste, ou: suite des Lettres écrites de Lausanne, l'anonimato dell'autrice era stato tuttavia presto svelato. In questo caso, l'esitazione tra le due indicazioni parageneriche non metterebbe quindi in dubbio tanto la paternità del testo quanto il suo legame con l'opera precedente. Durante tutto il XVIII secolo, pratiche editoriali e di composizione differenti da quelle contemporanee rendevano possibile e anzi molto frequente la ripresa di un'opera, fosse questa terminata o meno. Se il termine «continuation» - che evoca già l'incompiutezza del testo precedente - ci sembra oggi più appropriata, durante tutto il Settecento il fatto di riprendere un romanzo «finito» non costituisce né un problema né un caso eccezionale. Riprendendo l'osservazione di Genette, il termine «continuation» implica che le Lettres écrites de Lausanne siano incomplete, il che non è del tutto esatto, poiché il romanzo è materialmente concluso. Il termine «suite» - termine scelto, lo ricordiamo, dall'autrice e che figura nell'edizione originale - risulta quindi più adeguato poiché suggerisce un rapporto diverso con l'opera precedente, la cui compiutezza o meno è non precisamente definita e, in ogni caso, considerata come ininfluente.

Esaminando T2 quale appare al momento della sua «nascita», cioè nell'edizione di Prault del 1787, risulta inoltre chiaro che si tratta di un titolo vero e proprio. Tale precisazione si rende necessaria per sfatare ogni ambiguità eventualmente dovuta alla presentazione dell'enunciato così come compare in numerose edizioni ${ }^{40}$, ivi compresa quella delle Euvres complètes. Gli editori di quest'ultima hanno ripreso un'altra

(38) Il titolo dell'edizione originale, pubblicata nel 1787 da Prault, recita: CALISTE, OU: SUITE DES LETTRES ECRITES DE LAUSANNE. Nella seconda edizione di Prault, apparsa sempre nel 1787, il titolo appare già così modificato: CALISTE, OU CONTINUATION DES LETTRES ÉCRITES DE LAUSANNE. SECONDE PARTIE. Le edizioni successive riportano: CALISTE OU SUITE DES LETTRES ÉCRITES DE LAUSANNE. SECONDE PARTIE. (Prault, 1788); LETTRES ECRITES DE
LAUSANNE/PREMIERE PARTIE. (Prault, 1788, un'edizione che riunisce per la prima volta le due parti in un solo volume). Seguono altre edizioni ottocentesche, che modificano ulteriormente l'enunciato. Cf. I. De Charriere, Euvres complètes, cit., vol. VIII, pp. 615-616.

(39) Genette è tuttavia cosciente che «la distinction théorique se brouille assez souvent dans les faits». G. GentTte, Palimpsestes..., cit., pp. 181 sg.

(40) Cf. supra. 
edizione di Prault del 1787, successiva a quella originale, che presenta insieme i due romanzi di Madame de Charrière. T2 è citato solo in qualità di «seconde partie» delle Lettres écrites de Lausanne e non ha una sua pagina di titolo. Quest'ultima compare solo prima della prima parte delle Lettres écrites de Lausanne e non menziona il secondo enunciato ${ }^{41}$. T2 appare quindi nella prima pagina della «Seconde Partie» delle Lettres succitate precedendo la dicitura «Lettre XVIII» ${ }^{42}$. L'indicazione «Seconde Partie», così come la numerazione delle lettere, che riduce la prima lettera di Caliste alla diciottesima delle Lettres écrites de Lausanne, suggerisce una continuità tra le due parti di quella che è considerata una stessa opera. Inoltre, T2, così come appare nelle Euvres complètes, presenta le leggere modifiche citate precedentemente e riportate anche da altre edizioni settecentesche ${ }^{43}$. Stando a tale presentazione, esso non sarebbe più da considerarsi come un titolo vero e proprio, bensì come il titolo di una seconda parte. Riprendendo la terminologia di Genette, come un intertitolo ${ }^{44}$. È possibile considerare le due storie in questo modo. La pagina di titolo dell'edizione originale cita tuttavia l'enunciato per intero, scevro di indicazioni quali «seconde partie», restituendo pienamente a T2 la sua dimensione di titolo a tutti gli effetti.

Se lo consideriamo così come appare al momento della sua nascita, l'enunciato identifica dunque in modo univoco il testo, che descrive letteralmente. I rapporti tra le due parti di T2 sono precisamente definibili: si tratta di un enunciato doppio, formato da un titolo principale tematico - indica l'agente della diegesi ${ }^{45}$ e da uno secondario rematico, costituito a sua volta da un'indicazione generica inglobata nel titolo secondario - «suite des Lettres» - che rematizza il resto dell'enunciato. In questo caso anche T1 - titolo misto, come abbiamo visto precedentemente - viene completamente rematizzato. L'indicazione generica indica chiaramente che il romanzo in questione è la continuazione dell'opera intitolata Lettres écrites de Lausanne, il che esclude ogni tentazione di interpretazione tematica della seconda parte dell'enunciato. A rigor di logica, non potrebbe essere altrimenti. Il romanzo cui T2 rimanda termina con la partenza da Losanna delle due protagoniste, cosicché un'eventuale continuazione non potrebbe, in ogni caso, essere «écrite de Lausanne». Ma non solo. In Seuils, Genette sottolinea l'importanza acquisita da un'indicazione generica espressamente attribuita al testo dall'autore. In T2, l'indicazione generica mostra chiaramente che nonostante le ambiguità di un'opera che non è, come abbiamo già ricordato, propriamente una «suite» a livello tematico, il lettore deve considerare $C a$ liste, ou: suite des Lettres écrites de Lausanne come tale. Dietro preciso suggerimento di Isabelle de Charrière, il romanzo dovrebbe quindi essere letto come un'opera autonoma - essendo una «suite» e non una «seconde partie» - che mantiene uno stretto rapporto con il testo citato $^{46}$. Tale precisazione non è secondaria poiché influenza in un senso o nell'altro la lettura.

(41) I. De CHARRIÈre, Euvres complètes, cit., vol. VIII, p. 135.

(42) Ibid., p. 183.

(43) Caliste, ou continuation des Lettres écrites de Lausanne (Cf. Ibid., pp. 615-616).

(44) Il critico considera come intertitoli i titoli che si trovano «à l'intérieur des livres: titres de parties, de chapitres, de sections etc.» G. GENETTE, Seuils, cit., p. 106.

(45) Anche in questo caso valgono le precisazioni apportate da Genette stesso a proposito dell'aggettivo «tematico» e della sua leggera imprecisione.

(46) L'ambiguità della relazione tra Caliste, ou: suite des Lettres écrites de Lausanne e le Lettres écrites de Lausanne è stata più volte sottolineata dalla critica. P. Godet ha sottolineato i rapporti strettissimi che intercorrono tra le due parti: «Dès 1785 Mme de Charrière méditait la seconde partie du roman, la douloureuse histoire de Caliste. Caliste n'est donc pas - et l'on s'en doute bien à la lire - une «suite» imaginée après coup; il $\mathrm{y}$ a un lien intime entre les deux parties de l'ouvrage; elles s'expliquent, se complètent si heureusement, qu'on ne peut les séparer, et, bien que la seconde n'ait paru que deux ans après la première, elles furent manifestement conçues à la fois», op. cit., p. 30. $\grave{\mathrm{E}}$ difficile sapere se Caliste fu concepita contemporaneamente alle Lettres écrites de Lausanne. L'autrice pensò sicuramente molto presto a una suite del suo testo, ma tale intenzione resta poco espressa: in 
T2 è infine molto interessante a livello teorico poiché intrattiene importanti legami sia con il testo di Madame de Charrière già citato sia con opere di altri autori. E utile a questo proposito riprendere il concetto di «intertitolarità» introdotto da Hoek. Il critico precisa che

le titre doit se conformer au modèle général et en même temps il doit individualiser le co-texte et le distinguer des autres exemplaires de la série: l'intitulation se fait grâce à une différence dans l'imitation [...] on retrouve un paradoxe du titre: il réalise la clôture du texte et en même temps il ouvre le texte à d'autres discours par l'intertitularité [...] l'intertitularité permet de relier le modèle du titre à son environnement historique, social et culturel; cet espace social constitue une base pour rendre compte de l'évolution historique des titres ${ }^{47}$.

Anche da questo punto di vista, T2 presenta aspetti interessanti: formato da un nome proprio e da un titolo secondario rematico, l'enunciato rimanda direttamente a una struttura classica, mentre il termine «Lettres» evoca più precisamente il XVIII secolo. Prendendo in considerazione i rapporti tra $C$ e altri titoli e/o testi, è facile notare come un'intertitolarità multipla carichi l'enunciato di un forte valore connotativo. Senza contare l'intertitolarità tipologica e generica che abbiamo già evocato precedentemente a proposito di T1, ponendosi come «suite de» il titolo principale rinvia esplicitamente all'opera di Madame de Charrière citata nel titolo secondario e implicitamente ad altri testi precedenti. Si può riconoscere un'intertitolarità esterna sia implicita che esplicita. Infatti, l'adozione di un antroponimo femminile quale titolo principale rimanda, durante tutto il secolo, al genere del romanzo sentimentale, al quale anche il titolo $C$ accenna implicitamente. Più esplicitamente, questa parte dell'enunciato allude a testi precedenti, quali la pièce The fair penitent di Nicholas Rowe, o ad altri adattamenti francesi che riprendono il nome «Caliste» nel titolo ${ }^{48}$. La tragedia di Rowe ${ }^{49}$, adattata da The Fatal Dowry di Massinger, fu rappresentata per la prima volta a Londra nel maggio del 1703, ottenendo un grande successo ${ }^{50}$. Nel

nessun Avis dell'editore o in nessun altro elemento paratestuale Madame de Charrière annuncia la possibilità di una continuazione, e la menzione «Première Partie» appare per la prima volta solo nella pagina di titolo dell'edizione di Prault del 1787. Cf. Notes aux Lettres écrites de Lausanne, in Euvres complètes, VIII, p. 615. Il legame tra le due parti resta comunque molto forte, a livello tematico se non diegetico, come hanno sottolineato, tra l'altro, J. Starobinski e S. Minier. Cf. J. Starobinski, Les Lettres écrites de Lausanne de Madame de Charrière: inbibition psychique et interdit social, dans Roman et lumières au XVIIIe siècle, Paris, Éditions sociales, 1970, p. 133; S. MinIER-BIRK, Madame de Charrière. Les premiers romans, Paris-Genève, Slatkine, 1987, p. 55 .

(47) L. Hoek, op. cit., p. 185. Riprendendo i parametri di analisi della citazione, Hoek distingue intertitolarità interna - il titolo costituisce il testo citante e il co-testo quello citato - ed esterna, che può essere a sua volta ristretta - il rinvio si limita a una corrente letteraria, un genere o un autore - o più generale, esplicita o implicita. In generale, ogni titolo riflette un'intertitolarità tipologica, rinviando a un tipo di testo indipendentemente dal suo co-testo: «la lecture d'un texte ne se fait jamais à partir de zéro, rien que par la référence du titre à des modèles précédents. Tout titre connote un type d'écriture, une histoire de la littérature dans laquelle il s'insère, un genre auquel il appartient, un médium par lequel il s'exprime $[. .$.$] une période$ historique où il est produit et reçu, et une idéologie dans laquelle il s'inscrit». Ibid., p. 189.

(48) Il riferimento alla pièce inglese non è casuale. L'eroina di Madame de Charrière deve infatti il suo nome al personaggio di Rowe, che ha interpretato con grande successo una sola volta, prima di essere «ceduta» dalla madre a Lord **.

(49) $\mathrm{Si}$ tratta di una pièce in versi: Sciolto promette in sposa ad Altamont la figlia Calista nonostante quest'ultima ami Lothario. Scoperta la passione della sua futura moglie, Altamont uccide in duello il suo rivale. Accusandosi di essere la causa di tanta infelicità, Calista si suicida dopo essere stata perdonata dal padre, a sua volta colpito a morte dagli amici di Lothario.

(50) Il testo di Rowe, supportato da un cast eccezionale, ottenne un buon successo di pubblico e di critica, imponendo i personaggi di Calista e Lothario: «The playgoing public emphatically approved its pathos. The villain, 'the gallant, gay Lothario' acquired a proverbial reputation. The heroine, Calista, was a favourite character with the chief actresses of the century. Rowe's Lothario and Calista suggested Lovelace and Clarissa Harlowe to Richardson», Dictionary of National Biography, Vol. XVII, L. STHEPEN - S. LEE ed., Oxford, Oxford University Press, 1917, p. 342. 
1750, la traduzione francese di P. A. La Place doveva far conoscere l'autore e il testo anche fuori dai confini inglesi ${ }^{51}$. Come suggerisce P. Godet, Madame de Charrière potrebbe aver assistito a una rappresentazione inglese o essere venuta a conoscenza di adattamenti francesi quali la traduzione succitata Caliste ou la belle pénitente, o la Caliste di Colardeau, due pièces rappresentate alla Comédie Française intorno alla metà del secolo ed entrate a far parte di una tradizione letteraria $a^{52}$. Poiché rimanda esplicitamente ad un altro testo, il titolo principale di T2 costituisce dunque una citazione: citazione letterale nei confronti di Caliste, letterale e parziale rispetto a Caliste ou la belle pénitente, referenziale rispetto a The fair penitent. Il richiamo intertitolare e intertestuale carica l'enunciato di un forte valore connotativo e anche la scelta dell'antroponimo, in sé fortemente stereotipata ${ }^{53}$, acquista in tal modo una grande importanza. La pièce di Rowe, così come i suoi adattamenti francesi, tratta di un amore sfortunato e termina con la morte dei due amanti. «Caliste» si fa quindi segno ipersemantico: il nome proprio non solo anticipa l'identità dell'agente della diegesi, introduce un elemento di familiarità e designa un'ambientazione inglese, ma connota anche il genere e il soggetto del testo, anticipandone perfino la conclusione disforica. In teoria «Caliste», come ogni altro nome proprio, dovrebbe essere semanticamente neutro e non assumere alcuna connotazione morale, sociale o economica. In pratica, il nome proprio fictionnel acquisisce un valore non convenzionale grazie al rinvio esplicito ad un personaggio extratestuale. La valenza pragmatica del nome proprio di finzione, generalmente imprevedibile, è in questo caso accresciuta da quella connotativa attribuita dall'intertitolarità. Fungendo da nome proprio del testo, T2 lo designa e identifica, e allo stesso tempo ne riassume e ne esprime l'ideologia.

Anche l'indicazione paragenerica «suite» acquisisce una valenza connotativa, pur se meno esplicita, suggerendo la forma epistolare del testo. Analizzando i rapporti tra titolo principale e titolo secondario, possiamo notare che quest'ultimo rinvia al primo e di là indirettamente al testo, di cui fornisce un'informazione generica aggiuntiva. Allo stesso tempo, tale riformulazione costituisce un commento metalinguistico. Nel caso di T2, la dimensione metalinguistica è fortemente rimarcata. Non solo si può constatare la presenza di due indicatori tipografici - la virgola e i due punti -, che accentuano il carattere di enunciazione e riformulazione, ma il titolo secondario è

(51) Enciclopedia dello spettacolo, vol. VIII, Roma, Le Maschere, 1961, p. 1292; C. D. BRENNER, Bibliographical list of plays in the French language 1700-1789, New York, Ams Press, 1979, (ristampa dell'edizione di Berkeley, California, 1947), p. 152.

(52) «The Fair Penitent fut imité plusieurs fois. On possède Caliste ou la Belle pénintente, tragédie imitée de l'anglais; représentée pour la première fois sur le théâtre de la Comédie française le lundi 27 mars 1750. A Paris, chez Cailleau. MDCCL. [...] Selon les Anecdotes dramatiques, cette pièce, attribuée à l'abbé Séran de la Tour, était en réalité du marquis de Mauprié. Mlle Gaussin (la Zaïre de Voltaire) créa le rôle de Caliste. [...] En 1750, Colardeau fit jouer Caliste, tragédie en vers en 5 actes. Les personnages portent les mêmes noms que ceux des deux précédentes pièces. C'est Mlle Clairon qui tint alors le rôle de Caliste. Citons enfin la Lénore d'Andrieux, adaptation de la même donnée, qui eut, comme on voit, une assez longue fortune» $\mathrm{P}$. Godet, Madame de Charrière et ses amis, Genève, Slatkine Reprints, 1973 (réimpression de l'édition de Genève, 1906), pp. 321-322. Nella sua Biblio- graphical list, Clarence D. Brenner offre ulteriori precisazioni. Il critico presenta infatti Caliste ou la Belle pénitente come una traduzione di A. La Place della pièce di Rowe, citando tuttavia anche Mauprié e Séran de la Tour come possibili autori. La tragedia di La Place fu rappresentata per la prima volta alla Comédie Française il 27 maggio 1750 e pubblicata lo stesso anno, mentre secondo Brenner la Caliste di Colardeau fu messa in scena per la prima volta alla Comédie Française il 12 novembre 1760. Esistono inoltre un'altra Caliste, tragedia in versi imitata dalla traduzione francese e attribuita al marquis de Thibouville e una parodia del testo di La Place: Caliste, commedia in due atti di F. de Rozoi, pubblicata nel 1762. C. D. BRENNER, op. cit., pp. 152, 49, 63, 85, 122.

(53) «Caliste» è un antroponimo fortemente stereotipato: secondo la tipologia stilata da Hoek, la maggior parte dei nomi propri femminili che compaiono nei titoli dei romanzi francesi del XIX ${ }^{\circ}$ secolo che costituiscono il suo corpus iniziano per «C», terminano per «E» e hanno come penultima vocale la «I». Cf. op. cit., p. 240. 
abbastanza esteso per riformulare in modo visibile il titolo principale. Se si considera la riformulazione come un commento metalinguistico rivolto dall'autore al lettore a proposito del suo stesso enunciato, l'autore impone quindi in modo molto forte un «contrat de lecture» ${ }^{54}$. Da questo punto di vista, in una struttura sintattica è possibile ritrovare l'esplicitazione di un'intenzione dell'artista. Nel caso specifico di T2 - così come risultava dalla significativa inserzione dell'indicazione generica osservata da Genette -, la struttura sintattica mostra come la ricezione e la lettura di Caliste: ou, suite des Lettres écrites de Lausanne debbano essere quindi collegate a quelle delle Lettres écrites de Lausanne. Si può ancora riscontrare in T2 il carattere bivalente che Fromilhague attribuisce ad alcuni titoli narrativi di fine XVIII secolo ${ }^{55}$. Anche T2 offre infatti al lettore la possibilità di un «double programme de lecture $»^{56}$, che associa narrativo e non narrativo, destino individuale e preoccupazione descrittivoesplicativa. Se rinvia connotativamente al genere del romanzo sentimentale, Caliste, ou: suite des Lettres écrites de Lausanne esprime al tempo stesso l'ambiguità riscontrabile ugualmente in T1 - cui rimanda esplicitamente e di cui si pone come «suite» - annunciandosi anche come un testo descrittivo.

Per quanto riguarda l'aspetto pragmatico, quanto abbiamo detto a proposito di T1 resta valido anche nel caso di T2. Possiamo solo aggiungere che esiste un rapporto metonimico supplementare tra il titolo principale e le Lettres écrites de Lausanne, cioè l'opera cui il titolo secondario fa riferimento, che acquista per ciò stesso veridicità. Vero e proprio «titre-citation», T2 intrattiene dunque molteplici richiami intertestuali, che possono essere percepiti o meno a seconda della competenza culturale del lettore. Se pienamente colti, essi rimandano rispettivamente a una storia d'amore contrastata e infelice che finisce tragicamente e alla narrazione di un romanzo precedente di Madame de Charrière. Tale richiamo accresce sicuramente il potere seduttivo del titolo, facendo doppiamente leva sulla curiosità del pubblico, desideroso di conoscere la «fine» delle Lettres écrites de Lausanne - rimaste incompiute a livello strutturale e diegetico se non a livello materiale - e di cogliere grazie alla lettura del romanzo il nesso logico tra le due parti dell'enunciato, nesso esistente ma poco evidente a una prima occhiata. L'apparente mancanza di relazione logica tra l'elemento tragico evocato dal titolo principale e la storia delle Lettres écrites de Lausanne acuisce ancora di più la curiosità del lettore. Il grado di seduzione del titolo preso in esame è tuttavia relativo poiché soggettivo e strettamente legato alla comprensione dei richiami intertestuali citati. Pur con queste premesse, T2 può essere considerato come un titolo abbastanza «catchy» ${ }^{57}$ alla fine del XVIII secolo, grazie agli elementi «suite de» e «lettres de» e ai forti richiami intertestuali presumibilmente conosciuti da un tipo di pubblico socialmente connotato qual era l'élite culturale destinataria dei romanzi sentimentali della seconda metà del secolo.

I due enunciati presi in esame, a prima vista scontati e banali, si sono rivelati molto interessanti sia a livello teorico che dal punto di vista semantico-sintattico. Non solo è stato possibile ritrovarvi numerose chiavi di lettura per una migliore comprensione dei testi cui fanno riferimento, ma si è potuto constatare come la singolarità dei testi sia effettivamente già annunciata nei titoli, che anticipano alcuni elementi originali conferiti da Madame de Charrière a due romanzi che si distaccano dalla tradizione settecentesca dei «romans de femmes». I due testi in questione trovano in questa tradizione la loro naturale collocazione, poiché ne riprendono strutture formali - la

(54) C. Fromilhague, 'Titre principal' ou 'Titre secondaire'..., in A plus d'un titre..., cit., p. 132.

(55) Ibid., p. 138.
(56) Ibid.

(57) G. Genette, Seuils, cit., p. 98. 
modalità epistolare -, temi e topoi. La loro originalità consiste in una parziale sovversione di questi stessi codici, sovversione che si può riscontrare a livello di procedimenti formali - l'incompiutezza del primo testo o l'adozione di una scrittura e di una narrazione ambigue, «anfibie», che destabilizzano le certezze del lettore - e a livello tematico. Allo stesso modo, se T1 e T2 aderiscono ad una tradizione - quella delle «Lettres de...» - cui sono immediatamente riconducibili, da questa si distaccano a livello sintattico e semantico. È quindi possibile riscontrare come anche nel caso di enunciati apparentemente semplici e poco seduttivi agli occhi di un lettore contemporaneo quali quelli presi in esame, il titolo funzioni da specchio dell'opera, rivelando molto più di quanto potrebbe apparire ad una prima lettura. Opportuna e giustificata ci sembra perciò l'osservazione di M-H. Prat, che nelle sue Conclusions? del volume A plus d'un titre suggeriva che la risposta alla celebre domanda - «par où commencer?»- posta da R. Barthes a proposito dell'analisi di un'opera letteraria potesse e/o dovesse essere «par le titre $\rangle^{58}$. Quest'ultimo deve infatti essere considerato come la prima carta da visita, e non solo estetica, di un volume. Più o meno impostore, più o meno rivelatore, un buon titolo deve sicuramente, per riprendere la felice formula di U. Eco, «imbrogliare le idee». Tuttavia, per quanto «imbrogli», cioè «aggrovigli», «ingarbugli», le idee del lettore, l'enunciato non riesce mai ad «ingannarle» completamente. Al contrario, esso si fa sempre specchio del testo, costituendo così un punto privilegiato e diremmo quasi obbligato da cui cominciare l'analisi letteraria.

PAOLA PERAZZOLO 